\title{
Do pandemics justify non-evidence-based therapies?
}

\section{Luís Cláudio Lemos Correia' ${ }^{1}$ John Mandrola2 (1)}

\author{
${ }^{1}$ Corresponding author. Centre for Evidence-based Medicine, Escola Bahiana de Medicina e Saúde Pública (Salvador). Bahia, Brazil. \\ luis.correia@bahiana.edu.br \\ ${ }^{2}$ Division of Cardiac Electrophysiology, Baptist Health (Louisville). Kentucky, United States of America. john.mandrola@gmail.com
}

In hospitals both in the United States and in Brazil, leaders struggle to find the best way to monitor the QT interval of patients infected with the SARSCoV2 virus.

This is not because the virus affects cardiac ion channels but rather because doctorsall over the world-have ignored the tenets of the scientific method and evidence-based practice. While well-intentioned, the use hydroxychloroquine/azithromycin adds the potential for life-threatening pro-arrhythmia to patients with COVID-19.

In a shocking turn, one that we hope is merely coincidental, an accident of temporality, the announcement of President Donald Trump about the potential of Hydroxychloroquine for the treatment of COVID-19 preceded widespread embrace of this drug. The US president said: "We have to remove every barrier or a lot of barriers that were unnecessary and they've done that to get the rapid deployment of safe, effective treatments."
In this article, we consider decision making in a time of pandemic. Our ultimate goal is to transcend mere methodology and explore how doctors reason given the limits of scientific knowledge.

\section{When should the burden of proof of a treatment be dismissed?}

Not everything in Medicine requires empirical evidence. "Extreme plausibility" is real. Indeed, "parachute treatments" refer to a metaphorical situation in which the law of gravity makes the effectiveness of a parachute so obvious that a randomized clinical trial would be unethical ${ }^{1}$.

Parachute-therapies are common. Examples include emergency cricothyrotomy in a patient with airway obstruction, ventricular defibrillation, and blood transfusion in hemorrhagic shock with severe anemia. Here, empiricism is foolhardy. 
It is obvious that hydroxychloroquine/azithromycin for COVID-19 does not fulfill the parachute paradigm. But one could consider a more flexible version of extreme plausibility: "inexorable prognosis." In a situation of unavoidable fatality, the adoption of an unproven treatment may be applicable if an intermediate effect is certain.

For instance, while we don't know whether a mechanical ventricular assist device lowers mortality in patients with extreme circulatory failure, we do know that it improves hemodynamics. The rational for considering inexorable prognosis a version of extreme plausibility is that the probability of a beneficial effect may be low, but it is definitely higher than the probability of survival.

While we don't exactly know the infection fatality rate $^{2}$, we know COVID-19 is surely not a disease of inexorable prognosis. And hydroxychloroquine does not offer any guaranteed intermediate effect. Thus, doctors ought to have proof of efficacy of this treatment before using it. As of now, there is no evidence of minimally acceptable quality indicating clinical efficacy of this drug for patients with COVID-19.

\section{Blindness of Probability and Unintended Consequences}

When we lower the bar of scientific proof in medical science, we ignore probability. Effective treatments translate to favorable prognostic probabilities. What clinicians actually offer to the patient is a probability. Even with therapies with a large average absolute risk reduction, there will always be a number needed to treat $>1$. In fact, most therapies have NNTs much higher than one, indicating that beneficial therapies are infrequently beneficial in individual terms.

Now consider a treatment of unknown clinical effect. The probability turns into a conditional: P1 (probability of individual benefit, low in the best cases) x P2 (pretest probability of a mere scientific hypothesis). Multiplying two probabilities results in a lower probability. This is the case of hydroxychloroquine and/or azithromycin. Worse, we also do not know the nature of the potential beneficial effect: do the drugs prevent death, complications, residual pulmonary disease or only time for recovery?
Scientific rigor is not a scientific technique, it is pragmatic thinking. Pragmatism calls for clinicians caring for patients with COVID-19 to balance the low probability of benefit effect against unintended consequences. The latter are multiple, varying from predictable ones (QT prolongation) to others never imagined. Unintended consequences have no simple conditional probability (P1 x P2). Rather, there are an unknown number of unintended consequences and the probabilities add up (P1 + P2 + P3 + P4 .....), resulting in a high probability of at least one adverse outcome occurring.

Adverse effects and drug interactions are possible with any drug and this one is no exception ${ }^{3}$. However, indirect consequences may be as bad. First is distraction or the dispersion of efforts and attention to futile behaviors, at the expense of a focused quality of care. Another is cognitive fatigue of medical teams with a heap of useless information, such as QT monitoring algorithms. Finally: FALSE hope and the political use of treatments is especially toxic in a climate of public fear.

\section{Differentiation between Systemic and Individual Risk}

The US president's observations that "in this critical situation, we cannot place scientific barriers" confuses the systemic problem of the pandemic with the individual problem of having an infection. Indeed, an epidemic is a systemic phenomenon that requires large scale measures ${ }^{4}$. But when a person acquires the disease from an epidemic, the problem turns from a systemic one into an individual disease.

Consider two patients. One is an older person with co-morbid conditions who has COVID-19. Another patient with similar co-morbidities has bacterial sepsis. The fact that one person was infected during a viral pandemic and the other was not should not bear on evidence-based rationality. If we accept hydroxychloroquine/azithromycin for COVID-19 despite no proof of efficacy, we must accept vitamin $C$ for sepsis. Both are treatments that have being proposed but lack proof of efficacy ${ }^{5,6}$. 


\section{Conclusion}

Scientific integrity serves society as the means for the ultimate purpose of science: create valuable knowledge and solutions to human problems. Medicine is not science in itself. Its ultimate purpose is improving clinical outcomes and making people feel better. To reach this goal, medicine should be based on good quality scientific knowledge.

In moments of despair, medical rituals provide mental support to families and patients. It is part of our job. As part of the art of medicine, good physicians have their own subtle medical rituals. Prescribing a potentially harmful drug of unproven and unlikely efficacy may not the best ritual.

\section{Author contributions}

Both authors wrote and approved the final version of the paper.

\section{Competing interests}

No financial, legal or political competing interests with third parties (government, commercial, private foundation, etc.) were disclosed for any aspect of the submitted work (including but not limited to grants, data monitoring board, study design, manuscript preparation, statistical analysis, etc.).
1. Hayes MJ, Kaestner V, Mailankody S, Prasad V. Most medical practices are not parachutes: a citation analysis of practices felt by biomedical authors to be analogous to parachutes. CMAJ Open. 2018;6(1):31-38. doi: 10.9778/cmajo.20170088

2. Ioannidis JPA. Coronavirus disease 2019: The harms of exaggerated information and non-evidence-based measures. Eur J Clin Invest. 2020;50:e13222. doi: 10.1111/eci.13222

3. Chatre C, Roubille F, Vernhet H, Jorgensen C, Pers Y-M. Cardiac Complications Attributed to Chloroquine and Hydroxychloroquine: A Systematic Review of the Literature. Drug Saf. 2018;41(10):919-931. doi: 10.1007/s40264-018-0689-4

4. Maharaj S, Kleczkowski A. Controlling epidemic spread by social distancing: do it well or not at all. BMC Public Health. 2012;12:679. doi: $10.1186 / 1471-2458-12-679$

5. Pacheco RL, Riera R. Hydroxychloroquine and chloroquine for COVID-19 infection. Rapid systematic review. J. Évid-Based Healthc. 2020;2(1). doi: 10.17267/2675-021Xevidence.v2i1.2843

6. Fujii T, Luethi N, Young PJ, Frei DR, Eastwood GM, French CJ et al. Effect of Vitamin C, Hydrocortisone, and Thiamine vs Hydrocortisone Alone on Time Alive and Free of Vasopressor Support Among Patients With Septic Shock: The VITAMINS Randomized Clinical Trial. JAMA. 2020;323(5):423-431. doi:10.1001/jama.2019.22176 